\title{
Análise dos tempos entre chegadas de lotes em uma indústria alimentícia
}

\author{
Analysis of the times between arrivals of batches in a food industry \\ Claudio Luis Piratelli ${ }^{1} \quad$ Jorge Alberto Achcar ${ }^{1,2} \quad$ Renata Regina Sandrim ${ }^{1}$ \\ Recibido 19 de julio de 2012, aceptado 13 de enero de 2015 \\ Received: July 19, 2012 Accepted: January 13, 2015
}

\begin{abstract}
RESUMO
Em geral, a identificação das distribuições de probabilidades dos tempos entre chegadas, bem como das covariáveis que os afetam é tratada de forma superficial nos artigos sobre engenharia de produção. O objetivo principal deste trabalho é analisar os dados sobre os tempos entre chegadas de lotes de receitas ao departamento de controle de qualidade de uma indústria alimentícia. Para a modelagem estatística -abordagem quantitativa- utilizou-se uma distribuição de Weibull. Os parâmetros de escala e forma da distribuição foram estimados pelo método da máxima verossimilhança. Um modelo de regressão de Weibull foi apresentado para a avaliação de inferências nos parâmetros tempos médios e medianos entre chegadas e, para detectar os fatores que afetam a variabilidade dos dados coletados. Na presença de algumas covariáveis, observou-se que a distribuição de Weibull teve boa aderência aos dados. Os resultados permitiram uma modelagem mais fidedigna do setor de controle de qualidade da empresa para a realização de simulação discreta de eventos.
\end{abstract}

Palavras chave: Tempos entre chegadas, modelagem estatística, distribuição de Weibull, modelos de regressão, indústria.

\begin{abstract}
In general, the identification of probability distributions for time between arrivals and the covariates that affect them, get a superficial treatment in papers on production engineering. The main goal of this paper is to analyze the data on the time between arrivals for batches at the quality control department in a food company. A Weibull distribution, with two parameters, was used in the statistical modeling - a quantitative approach. Scale and shape was estimated by the Maximum Likelihood Method. A regression Weibull model was considered to evaluate inferences for the mean and median times between arrivals and to detect important factors affecting the variability in data sets. Considering some covariates, the Weibull distribution was found to have a good fit for the data. These results allow for a more reliable modeling of the quality control department for discrete event simulation.
\end{abstract}

Keywords: Time between arrivals, statistical modeling, Weibull distribution, regression models, industry.

\section{INTRODUÇÃO}

Variações em processos de chegadas são observadas em diversas situações práticas onde a taxa de chegada de entidades é fortemente dependente do tempo. Na indústria, a modelagem estatística destes dados é imprescindível para escolha de um modelo de Filas adequado ao sistema produtivo que

\footnotetext{
1 Engenharia de Produção. Centro Universitário de Araraquara. Rua Voluntários da Pátria, 1295 - Centro, CEP: 14801-320. Araraquara-SP, Brasil. E-mail: clpiratelli@uniara.com.br; renatasandrim@ hotmail.com

2 Faculdade de Medicina de Ribeirão Preto. Universidade de São Paulo (USP). Av. Bandeirantes, 3900 - Monte Alegre, CEP: 14049-900. Ribeirão Preto-SP, Brasil. E-mail: achcar@fmrp.usp.br
} 
se pretende diagnosticar (determinar indicadores de desempenho) ou realizar experimentos por meio de Simulação Discreta de Eventos. Estas duas abordagens da Pesquisa Operacional permitem que os gestores tomem melhores decisões sobre investimentos, programação da produção, alocação de capacidade produtiva etc. Além disso, a descoberta de possíveis fatores que atuam nessas variações pode ser de grande interesse para engenheiros e administradores industriais.

Na prática, dois tipos de dados são fundamentais para a modelagem estatística de sistemas produtivos: as contagens das unidades produzidas por unidade de tempo ou os tempos entre as chegadas destas unidades. Para modelar os primeiros, uma possibilidade é assumir processos de Poisson homogêneos ou não-homogêneos [1-4]. Para os segundos, uma alternativa é coletar e analisar estatisticamente os tempos entre chegadas das unidades denotados por

$$
t_{i}=x_{i}-x_{i-1}
$$

onde $i=1,2, \ldots, n$ denota a i-ésima unidade; $x_{i}$ denota o horário de chegada i-ésima unidade; $x_{i}$ denota o horário de chegada da unidade antecessora, e; n é o número de unidades recebidas durante um determinado período de tempo.

O objetivo principal deste trabalho é analisar os dados sobre tempos entre chegadas de lotes de receitas ao departamento de controle de qualidade de uma indústria do setor alimentício do interior do estado de São Paulo, Brasil. Para isso será utilizada a distribuição de Weibull e seus parâmetros de escala e forma serão estimados pelo método da máxima verossimilhança, visando propiciar ao analista em Simulação Discreta de Eventos da indústria, uma modelagem mais fidedigna aos dados coletados. Um modelo de regressão de Weibull também será apresentado para a avaliação de inferências nos parâmetros tempos médios e medianos entre chegadas $\mathrm{e}$, detectar os fatores que afetam a variabilidade dos dados coletados.

Metodologicamente este trabalho pode ser classificado como aplicado, de objetivo descritivo e abordagem quantitativa [5]. A pesquisa quantitativa em Engenharia de Produção é definida como aquela em que se modela um problema cujas variáveis apresentam relações causais e quantitativas [6].
Neste sentido, torna-se possível quantificar o comportamento das variáveis dependentes sob um domínio específico, permitindo ao pesquisador realizar predições. Em geral, as pesquisas quantitativas utilizam modelagem matemática, estatística ou computacional (simulação) - especificamente, neste trabalho será adotada a modelagem estatística. Quanto às técnicas de pesquisa serão utilizadas a pesquisa bibliográfica e a observação direta intensiva [7]; ou a pesquisa bibliográfica e o estudo de caso [8].

O artigo está organizado da seguinte forma: a seção 2 trata brevemente da importância da modelagem estatística dos tempos entre chegadas para estudos industriais e apresenta a distribuição de Weibull; a seção 3 apresenta a descrição do problema, as estatísticas descritivas dos dados coletados, a análise de aderência da distribuição de Weibull e seus parâmetros estimados aos dados coletados e, o modelo de regressão utilizado; finalmente, a seção 4 traz uma discussão dos resultados obtidos e as considerações finais.

Modelagem estatística de tempos entre chegadas

Em Teoria das Filas (QT), a análise estatística de tempos -tempos entre chegadas de entidades a um sistema e tempos de serviços-é fundamental para a escolha do modelo de fila adequado ao objeto que se pretende diagnosticar (identificar indicadores de desempenho como: utilização média dos recursos, número de entidades em fila, tempo médio de permanência das entidades no sistema etc.) - ver, por exemplo [9-11]. Diversos artigos da área de QT abordam a análise dos tempos entre chegada. Ver alguns exemplos: [12-20].

Em Simulação Discreta de Eventos um dos principais objetivos do analista é realizar experimentações em modelos que representam sistemas reais, como forma de prever comportamentos futuros. Neste sentido, a identificação das distribuições de probabilidades dos tempos (entre chegadas e serviços) e seus parâmetros é crucial para que se tenha um modelo fidedigno à realidade que se pretende modelar [21-23].

A etapa de identificar distribuições de probabilidades de tempos e seus parâmetros, muitas vezes é tratada de forma superficial em artigos que tratam do processo de modelagem em Simulação Discreta de Evento [24]. Neste sentido, o presente artigo 
contribui ilustrando como identificar os parâmetros da distribuição de Weibull, aderentes aos dados industriais de tempo entre chegadas de produtos a um departamento de controle de qualidade (o qual a indústria deseja diagnosticar).

\section{A distribuição de Weibull}

Para analisar os dados de tempos (tempos entre chegadas ou tempos de serviços) pode-se utilizar usar uma distribuição de probabilidade muito popular na área de confiabilidade industrial: a distribuição de Weibull com dois parâmetros [25-28].

A distribuição de Weibull tem sido muito utilizada na análise de dados médicos - ver, por exemplo, [29] - e industriais - ver, por exemplo, [30-33] dada a sua grande flexibilidade de ajuste [27 e 34].

A função densidade de probabilidade da distribuição de Weibull é dada por,

$$
f\left(t_{i}\right)=\frac{\alpha t^{\alpha-1}}{\lambda^{\alpha}} \exp \left\{-\left(\frac{t}{\lambda}\right)^{\alpha}\right\},
$$

onde $t_{i}>0$ denota os tempos entre chegadas dos lotes de receitas para o controle de qualidade. Os parâmetros $\lambda$ e $\alpha$ denotam respectivamente os parâmetros de escala e de forma. Diferentes valores de $\alpha$ levam a diferentes formas para a distribuição o que a torna muito flexível na análise de dados para tempos entre chegadas.

A função de confiabilidade para um tempo t* é dada por,

$$
R\left(t^{*}\right)=P\left(T>t^{*}\right)
$$

Observar que (3) representa a probabilidade de um tempo entre chegadas ser maior do que um valor fixado $t^{*}\left(t^{*} \geq 0\right)$. Assumindo a distribuição de Weibull descrita em (2), tem-se que,

$$
R\left(t^{*}\right)=\exp \left\{-\left(\frac{t^{*}}{\lambda}\right)^{\alpha}\right\}
$$

A função de risco h(t) ou taxa instantânea de falha, da distribuição de Weibull - ver, por exemplo, [27, 28 e 34] - é dada de $\mathrm{h}(\mathrm{t})=\mathrm{f}(\mathrm{t}) / \mathrm{R}(\mathrm{t})$ por,

$$
h(t)=\alpha t^{\alpha-1} / \lambda^{\alpha}
$$

Observar que se $\alpha=1$, temos a distribuição exponencial, que é um caso particular da distribuição de Weibull. A função de risco h(t) dada por (5), que mede o risco de falha, é estritamente crescente para $\alpha>1$ (isto é, o risco de falha aumenta com o tempo, numa linguagem de durabilidade industrial), estritamente decrescente para $\alpha<1$ (isto é, o risco de falha diminui com o tempo, numa linguagem de durabilidade industrial) e constante para $\alpha=1$. Assim, observa-se uma grande flexibilidade de ajuste aos dados. A média e a variância da distribuição de Weibull com densidade dada por (2) são dadas respectivamente por,

$$
\begin{aligned}
& \mu=E(T)=\lambda \Gamma\left(1+\frac{1}{\alpha}\right), e \\
& \sigma^{2}=\lambda^{2}\left\{\Gamma\left(1+\frac{2}{\alpha}\right)-\left[\Gamma\left(1+\frac{1}{\alpha}\right)\right]^{2}\right\},
\end{aligned}
$$

onde $\Gamma$.) denota uma função gama, $\Gamma(\mathrm{z})=\int_{0}^{\infty} e^{-t} t^{z-1} d t$
ver, por exemplo, [35].

Estimadores para os parâmetros $\lambda$ e $\alpha$ podem ser obtidos usando o método de máxima verossimilhança - ver, por exemplo, [36] - obtidos maximizando a função de verossimilhança $L(\lambda, \alpha)=\prod_{i=1}^{n} f\left(t_{i}\right)$.

. Considerando a distribuição de Weibull com densidade (2) a função de verossimilhança é dada por,

$$
L(\lambda, \alpha)=\prod_{i=1}^{n} \frac{\alpha t_{i}^{\alpha-1}}{\lambda^{\alpha}} \exp \left\{-\left(\frac{t_{i}}{\lambda}\right)^{\alpha}\right\}
$$

Com $n$ sendo o tamanho amostral (número de unidades). Os estimadores de máxima verossimilhança (EMV), em geral, são obtidos maximizando-se o logaritmo da verossimilhança, $l(\lambda, \alpha)=\log (L$ $(\lambda, \alpha))$ e dados pelas soluções das equações de verossimilhança,

$$
\begin{aligned}
& \frac{\partial l(\lambda, \alpha)}{\partial \lambda}=0, \\
& \frac{\partial l(\lambda, \alpha)}{\partial \alpha}=0
\end{aligned}
$$


As soluções das equações (8) são obtidas usando um método numérico iterativo, como por exemplo, o método de Newton-Raphson - ver [37]. Intervalos de confiança e testes de hipóteses de interesse são obtidos usando a normalidade assintótica dos estimadores de máxima verossimilhança (EMV) denotados por $\hat{\lambda}$ e $\hat{\alpha}$ - ver, por exemplo, [27].

\section{ESTUDO DE CASO}

A indústria alimentícia estudada localiza-se no interior do Estado de São Paulo e produz molho de tomate, mostarda, condimentos, enlatados (tais como ervilhas, milho, doces em conserva etc.). Cada tipo de produto possui um padrão específico garantido por sua receita. Operadores denominados "pontistas" são responsáveis por preparar cada receita e coletar amostras para serem analisadas no departamento de controle de qualidade (a empresa inspeciona $100 \%$ das receitas para que todos os produtos tenham qualidade assegurada). No departamento de controle da qualidade as amostras são analisadas por ordem de chegada - disciplina FCFS (first come first served) - por dois analistas aptos para inspecionar qualquer tipo de produto. Após a realização de testes físico-químicos e testes organolépticos as amostras aprovadas liberam as receitas para as linhas de envase.

A empresa trabalha com dois turnos de produção e o departamento de controle da qualidade funciona 10hs/dia. Os dados de intervalo entre chegadas de amostras das receitas ao departamento foram obtidos por meio de anotações, conforme (1), sobre os horários de chegadas em cada dia, durante um período de 30 dias produtivos consecutivos. As estatísticas descritivas destes dados são apresentadas na Tabela 1. A Figura 1 apresenta os Box-plots dos tempos médios entre chegadas diárias, elaborados através do software MINITAB versão14.

Pela Tabela 1 e pela Figura 1 é possível notar que há grande variabilidade nas contagens diárias de unidades (amostras de receitas) que chegam para inspeção de qualidade e a indústria tem interesse em identificar os fatores que afetam essa variabilidade, especialmente para uma modelagem mais real do departamento por meio de ferramentas de Simulação Discreta de Eventos. Ainda pelos Box-plots da Figura 1, pode-se observar que a distribuição para os tempos entre chegadas tem formas assimétricas.
Tabela 1. Estatísticas descritivas para $\mathrm{t}_{\mathrm{i}}(\mathrm{min}) \mathrm{de}$ receitas no departamento de controle de qualidade.

\begin{tabular}{|c|c|c|c|c|c|c|c|}
\hline :ै & Z & 窇 & بُ & 己 & 总 & & 总 \\
\hline 1 & 60 & 10,33 & 11,33 & 1,10 & 0,08 & 8,65 & 60,50 \\
\hline 2 & 94 & 6,42 & 6,90 & 1,07 & 0,03 & 3,83 & 33,85 \\
\hline 3 & 118 & 5,39 & 5,21 & 0,97 & 0,02 & 3,93 & 32,63 \\
\hline 4 & 109 & 5,43 & 6,15 & 1,13 & 0,03 & 3,35 & 39,00 \\
\hline 5 & 76 & 6,40 & 6,18 & 0,97 & 0,02 & 4,58 & 29,73 \\
\hline 6 & 63 & 9,94 & 12,41 & 1,25 & 0,05 & 6,00 & 80,47 \\
\hline 7 & 91 & 6,78 & 7,06 & 1,04 & 0,03 & 4,97 & 35,08 \\
\hline 8 & 95 & 6,48 & 9,91 & 1,53 & 0,03 & 3,73 & 82,27 \\
\hline 9 & 85 & 6,83 & 11,33 & 1,66 & 0,05 & 4,83 & 97,23 \\
\hline 10 & 81 & 6,54 & 6,50 & 0,99 & 0,15 & 4,67 & 35,45 \\
\hline 11 & 65 & 9,55 & 11,63 & 1,22 & 0,63 & 7,07 & 83,53 \\
\hline 12 & 82 & 7,71 & 6,24 & 0,81 & 0,17 & 6,49 & 39,45 \\
\hline 13 & 86 & 7,40 & 6,39 & 0,86 & 0,38 & 6,03 & 35,40 \\
\hline 14 & 88 & 7,05 & 7,16 & 1,02 & 0,10 & 5,68 & 54,97 \\
\hline 15 & 106 & 6,11 & 4,72 & 0,77 & 0,07 & 5,44 & 27,23 \\
\hline 16 & 115 & 5,66 & 4,60 & 0,81 & 0,20 & 4,38 & 28,60 \\
\hline 17 & 89 & 6,97 & 3,74 & 0,54 & 0,33 & 6,32 & 25,15 \\
\hline 18 & 100 & 6,29 & 4,15 & 0,66 & 0,52 & 5,37 & 20,38 \\
\hline 19 & 80 & 8,00 & 4,65 & 0,58 & 1,12 & 7,48 & 30,72 \\
\hline 20 & 81 & 7,99 & 4,38 & 0,55 & 1,48 & 7,93 & 25,88 \\
\hline 21 & 51 & 12,36 & 12,45 & 1,01 & 2,80 & 9,33 & 91,08 \\
\hline 22 & 54 & 11,98 & 9,42 & 0,79 & 4,48 & 11,43 & 76,23 \\
\hline 23 & 67 & 9,52 & 9,12 & 0,96 & 1,17 & 7,88 & 74,00 \\
\hline 24 & 71 & 9,11 & 6,26 & 0,69 & 0,47 & 7,47 & 29,62 \\
\hline 25 & 52 & 12,31 & 12,81 & 1,04 & 1,48 & 7,89 & 76,35 \\
\hline 26 & 63 & 10,00 & 10,53 & 1,05 & 1,30 & 7,57 & 80,05 \\
\hline 27 & 86 & 7,48 & 4,59 & 0,61 & 1,28 & 5,74 & 22,37 \\
\hline 28 & 102 & 6,33 & 5,20 & 0,82 & 0,50 & 4,82 & 35,35 \\
\hline 29 & 96 & 6,50 & 6,38 & 0,98 & 0,53 & 4,67 & 37,43 \\
\hline 30 & 86 & 7,03 & 6,81 & 0,97 & 0,85 & 5,54 & 43,63 \\
\hline
\end{tabular}

(N: observações por dia, CV: coeficiente de variação); observações com destaque são segundas-feiras; D. P. são desvios- padrões de $t_{\mathrm{i}}$.

\section{Análise para os Tempos entre Chegadas dos Lotes para o Controle de Qualidade}

Para a análise dos dados (tempos entre chegadas de amostras para o controle de qualidade), inicialmente considera-se a distribuição de Weibull com densidade (2). Usando o software MINITAB versão14, foram obtidas as estimativas de máxima verossimilhança assumindo todas as observações dos 30 dias (2492 receitas que chegam para o controle de qualidade).

A Tabela 2 apresenta os EMV, erros-padrões e intervalos de confiança $95 \%$ para os parâmetros $\alpha$ 


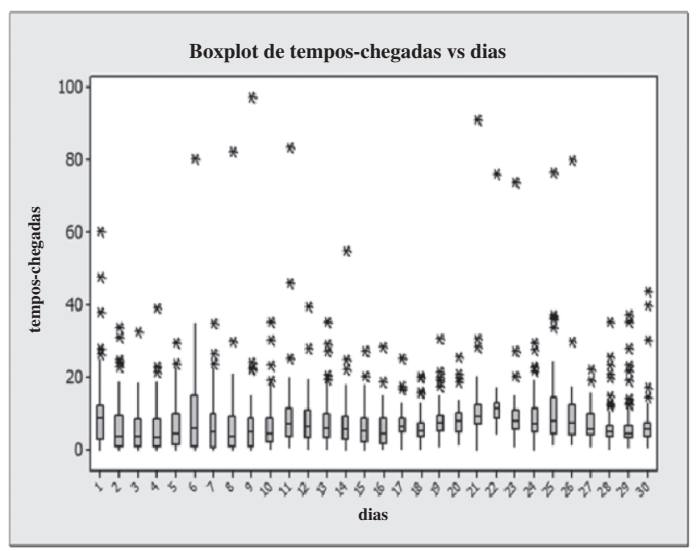

Figura 1. Box-plots para os tempos entre chegadas diárias.

e $\lambda$. Também apresenta as estimativas de máxima verossimilhança para o tempo médio entre chegadas dado por (6) e para o tempo mediano entre chegadas dado por $t_{\text {med }}=\lambda(\log 2)^{\frac{1}{\alpha}}$ (obtido igualando-se $R\left(t^{*}\right)=\exp \left\{-\left(\frac{t^{*}}{\lambda}\right)^{\alpha}\right\}$ a 0,5 , isto é, $\left.\mathrm{R}\left(\mathrm{t}_{\mathrm{med}}\right)=0,5\right)$.

Observar que o intervalo de confiança para o parâmetro de forma $\alpha$ não inclui o valor um, um indicativo de que a distribuição de Weibull é melhor ajustada aos dados, do que usando a distribuição exponencial onde o parâmetro de forma $\alpha$ é igual à um. A partir dos resultados dados na Tabela 2, é possível estimar as probabilidades dos tempos entre chegadas serem maiores do que valores fixados $t^{*}$. Estas estimativas são obtidas a partir de (4) com os valores de $\alpha$ e $\lambda$ dados pelas estimativas de máxima verossimilhança $\lambda=7,7338$ e $\alpha=1,0970$. A Tabela 3 apresenta as estimativas das probabilidades $\left(\hat{R}\left(t^{*}\right)=\exp \left\{-\left(\frac{t^{*}}{\hat{\lambda}}\right)^{\hat{\alpha}}\right\}\right)$ para alguns valores de $t^{*}$.

Tabela 2. Estimativas e intervalos de confiança para os parâmetros do modelo.

\begin{tabular}{|c|c|c|c|}
\hline Parâmetro & E.M.V. & E.P. & $\begin{array}{c}\text { Intervalo de Confiança } \\
95 \%\end{array}$ \\
\hline$\alpha$ & 1,0970 & 0,0164 & $(1,0653 ; 1,1296)$ \\
\hline$\lambda$ & 7,7338 & 0,1485 & $(7,4481 ; 8,0304)$ \\
\hline$\mu$ & 7,4692 & 0,1366 & $(7,2062 ; 7,7417)$ \\
\hline $\mathrm{t}_{\text {med }}$ & 5,5372 & 0,1178 & $(5,3110 ; 5,7731)$ \\
\hline
\end{tabular}

(E.P. = erro-padrão).

Fonte: própria.

Dos valores obtidos na Tabela 3, observa-se que há grande probabilidade dos tempos entre chegadas
Tabela 3. Estimativas de R ( $\left.\mathrm{t}^{*}\right)$ para alguns valores de $t^{*}$.

\begin{tabular}{|l|l|}
\hline $\mathbf{t}^{*}$ & $\hat{\boldsymbol{R}}\left(\boldsymbol{t}^{*}\right)$ \\
\hline 0,3208 & 0,9700 \\
\hline 0,7078 & 0,9300 \\
\hline 1,9705 & 0,8000 \\
\hline 5,5372 & 0,5000 \\
\hline 9,1597 & 0,3000 \\
\hline 16,5417 & 0,1000 \\
\hline 21,0263 & 0,0500 \\
\hline
\end{tabular}

Fonte: própria.

de lotes de receitas para controle de qualidade serem maiores do que 1,9705 (aproximadamente 2 minutos). Esta probabilidade é estimada por 0,80, isto é, espera-se que em $80 \%$ das vezes, os tempos entre chegadas sejam maiores do que 2 minutos. Da mesma forma, observa-se uma probabilidade muito pequena $(0,05)$ dos tempos entre chegadas serem maiores do que 21 minutos.

A distribuição de Weibull é bem ajustada pelos dados conforme pode se observar no gráfico de probabilidade Weibull da Figura 2.

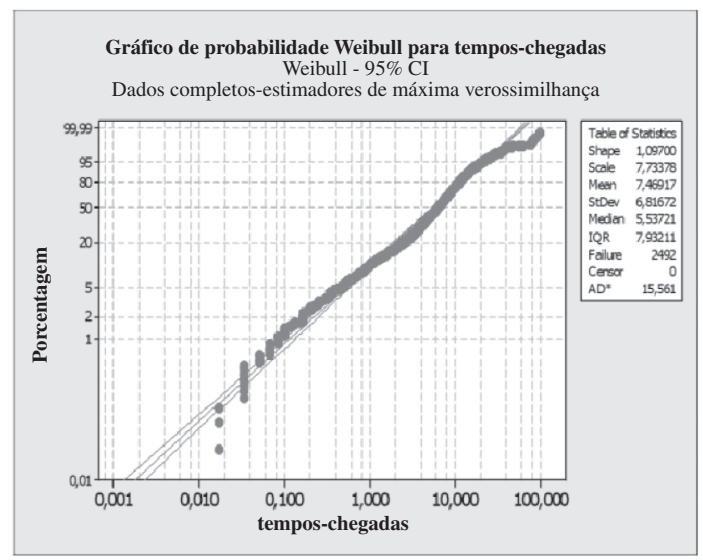

Figura 2. Gráficos de probabilidade (distribuição de Weibull).

Fonte: própria.

\section{Modelo de regressão}

$\mathrm{Na}$ análise de dados da Tabela 1 (estatísticas descritivas dos tempos entre chegadas de amostras para controle de qualidade) é possível observar que existe grande variabilidade para os tempos médios diários relacionados aos dias e alguma relação com os dias da semana, onde, aparentemente nas segundasfeiras os tempos médios entre chegadas são maiores 
o que indica contagens menores de unidades nas segundas-feiras. Uma possibilidade é considerar um modelo de regressão em (2) assumindo que só o parâmetro de escala $\lambda$ depende das covariáveis dias e indicador de dias e o parâmetro de forma $\alpha$ não depende das covariáveis.

Dessa forma pode-se assumir um modelo de regressão de valor extremo definido por,

$$
\begin{gathered}
\log \left(t_{i}\right)=\beta_{0}+\beta_{1} \text { dias }_{i}+\beta_{2} \text { ind }_{- \text {semanas }_{i}} \\
+\sigma^{*} \varepsilon_{i}
\end{gathered}
$$

onde $t_{i}$ são os tempos entre chegadas de unidades para o controle de qualidade; $\beta_{0}, \beta_{1}$ e $\beta_{2}$ são parâmetros de regressão; dias denotam os dias da semana (dias $_{\mathrm{i}}$ $=\mathrm{i}, \mathrm{i}=1,2, \ldots, 30$ ); ind - semanas $_{\mathrm{i}}$ denota (uma variável indicadora ou variável "dummy") o dia da semana, sendo ind - semanas $_{i}=1$ se o i-ésimo dia é uma segunda- feira e ind - semanas $_{\mathrm{i}}=0 \mathrm{se}$ o i-ésimo dia for um dia diferente de segundafeira. O parâmetro $\sigma^{*}$ está relacionado com o parâmetro de forma da distribuição de Weibull (2) pela relação $\sigma^{*}=1 / \alpha$ e o parâmetro de escala $\lambda$ dependente das covariáveis é dado pela relação $\lambda_{i}$ $=\exp \left(\beta_{0}+\beta_{1}\right.$ dias $_{i}+\beta_{2}$ ind - semanas $\left._{i}\right)$. O termo $\varepsilon i$ em (9) é uma quantidade aleatória com distribuição de valor extremo - ver [27] ou [37] - também definida como distribuição de valor extremo de tipo I (mínimo) ou distribuição de Gumbel [38] com função densidade de probabilidade dada por $\mathrm{f}(\varepsilon)=\exp (\varepsilon-\exp (\varepsilon)),-\infty<\varepsilon<\infty$. De (8) observa-se que $t_{i}=\exp \left(\beta_{0}+\beta_{1}\right.$ dias $_{i}+\beta_{2}$ ind- semanas $\left._{i}+\sigma * \varepsilon_{i}\right)$ isto é, um modelo de tempo de vida acelerado - ver, por exemplo, [37].

Para o modelo de regressão (9), também é possível estimar os parâmetros de regressão $\beta_{0}$, $\beta_{1}$ e $\beta_{2}$ e o parâmetro $\sigma^{*}$ usando métodos de máxima verossimilhança. Estimadores de máxima verossimilhança para os parâmetros $\beta_{0}, \beta_{1}, \beta_{2}$ e $\sigma^{*}$ são obtidos maximizando-se a função de verossimilhança

$$
L(\lambda, \alpha)=\prod_{i=1}^{n} f\left(\varepsilon_{i}\right) \text { onde } \mathrm{f}\left(\varepsilon_{i}\right)=\exp \left(\varepsilon_{i}-\exp \left(\varepsilon_{i}\right)\right),
$$

$\mathrm{i}=1, \ldots \mathrm{n}, \mathrm{e}$,

$$
\begin{aligned}
& \varepsilon_{i}=\left[\log \left(t_{i}\right)-\beta_{0}-\beta_{1} \text { dias }_{i}-\beta_{2}\right. \text { ind } \\
& \left.- \text { semanas }_{i}\right] / \sigma *
\end{aligned}
$$

Na prática, em geral maximiza-se o logaritmo da função de verossimilhança na determinação dos estimadores de máxima verossimilhança.

Tabela 4. Estimativas e intervalos de confiança para os parâmetros do modelo (modelo de regressão de valor extremo).

\begin{tabular}{|c|c|c|c|}
\hline $\begin{array}{c}\text { Parâ- } \\
\text { metro }\end{array}$ & EMV & E.P. & $\begin{array}{c}\text { Intervalo de } \\
\text { Confiança 95\% }\end{array}$ \\
\hline$\beta_{0}$ & 1,8574 & 0,0392 & $(1,7806 ; 1,9342)$ \\
\hline$\beta_{1}$ & 0,0083 & 0,0021 & $(0,0041 ; 0,0123)$ \\
\hline$\beta_{2}$ & 0,3042 & 0,0465 & $(0,2131 ; 0,3953)$ \\
\hline$\alpha$ & 1,1073 & 0,0166 & $(1,0752 ; 1,1403)$ \\
\hline
\end{tabular}

Fonte: própria.

A partir do modelo de regressão (9) com a presença das covariáveis dias e ind-semanas, apresenta-se na Tabela 4 os EMV, os erros-padrões e os intervalos de confiança $95 \%$ para os parâmetros $\beta_{0}, \beta_{1}, \beta_{2} \mathrm{e}$ $\alpha=1 / \sigma^{*}$ (parâmetro de forma da distribuição de Weibull).

Testes de hipóteses foram realizados para verificar a significância dos parâmetros de regressão $\beta_{1}$ e $\beta_{2}$ associados aos dias e aos dias-semanas. Da aproximação normal para os EMV de $\beta_{1}$ e $\beta_{2}$ determinou-se (através do software MINITAB versão14) os valores da estatística $Z$ (distribuição normal padronizada) dados respectivamente por $\mathrm{Z}=3,90$ (dias) e $\mathrm{Z}=6,55$ (dias-semanas) com respectivos p-valores menores do que 0,01 . Assim observa-se forte indicação do grande efeito das covariáveis dias e ind-semanas na variabilidade dos tempos entre chegadas dos lotes de receitas para o controle de qualidade, isto é, os valores de $\beta_{1}$ e $\beta_{2}$ são estatisticamente diferentes de zero.

Observar que $\beta_{2}$ é estimado por 0,3042 (um valor positivo). Apesar de não se ter uma interpretação direta para com a média dos tempos entre chegadas dos lotes para o controle de qualidade como é feito em modelos de regressão lineares usuais, pois a regressão considerada afeta o parâmetro de escala da distribuição de Weibull dado por $\lambda_{i}=\exp \left(\beta_{2}+\right.$ $\beta_{1}$ dias $_{i}+\beta_{2}$ ind-semanas ${ }_{i}$ ),observa-se que as médias $\mu i$ dada por (6), assumindo $\alpha$ fixo, seriam maiores para as segundas-feiras, pois ind - semanas $_{\mathrm{i}}=$ 1 se o i-ésimo dia é uma segunda- feira e ind semanas $_{\mathrm{i}}=0$ se o i-ésimo dia for um dia diferente de segunda-feira. 
A Figura 3 apresenta os gráficos dos gráficos de probabilidade dos resíduos padronizados do modelo de regressão (9) ajustado. Observa- se bom ajuste do modelo para os dados [37], pois os pontos estão bem próximos de uma relação linear (ver Figura 3). Outras distribuições usuais para dados de confiabilidade, como a distribuição log-normal também foram ajustadas, mas ganhos no ajuste (como os observados nos gráficos dos gráficos de probabilidade da Figura 3 ) não foram observados.

\section{DISCUSSÃO DOS RESULTADOS OBTIDOS E CONSIDERAÇÕES FINAIS}

As análises estatísticas empreendidas neste trabalho se mostraram eficazes para a identificação das causas da grande variabilidade entre os tempos entre chegadas de amostras para o controle de qualidade (nos diferentes dias). O uso da distribuição de Weibull levou à obtenção de inferências precisas para os parâmetros do modelo com especial destaque para os tempos médios e medianos entre chegadas. Além disso, com o uso do modelo ajustado foi possível fazer previsões, em termos probabilísticos, de que esses tempos ultrapassem determinados valores fixados. O uso de outras distribuições de probabilidade, como a distribuição log-normal não levou a melhores ajustes aos dados.

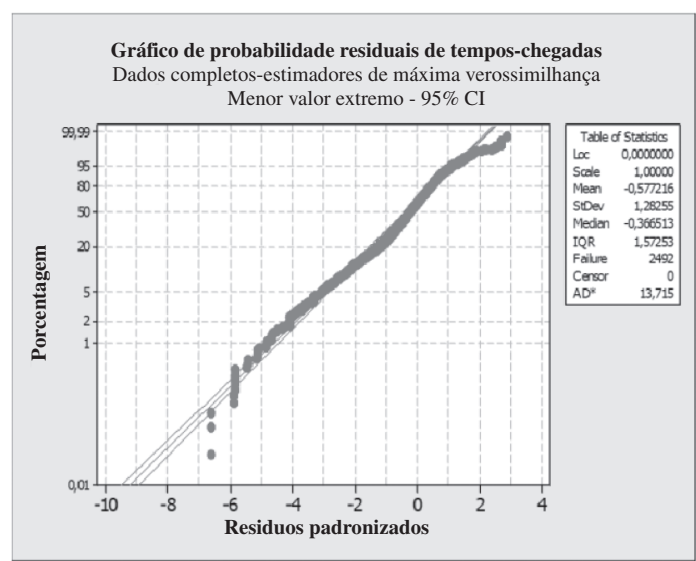

Figura 3. Gráficos de probabilidade dos resíduos padronizados.

Fonte: própria.

Com o modelo de regressão (9), foi possível detectar dois fatores que afetam a variabilidade dos dados: os dias e as segundas-feiras (dia da semana). Cabe ressaltar que seria factível incorporar nesse modelo outros fatores como índices econômicos, estações do ano, entre vários outros.

O dia da semana (segunda-feira ou não) tem grande influência na variabilidade dos dados: nas segundas-feiras o tempo médio entre chegadas é igual à 9,334 minutos e nos outros dias da semana essa média é igual à 7,030, com desvios-padrões dados respectivamente por 10,090 e 6,917 . Na Figura 4, apresenta-se os box-plots para os dados nas segundas-feiras e para os outros dias da semana. Observar que, considerando-se um modelo de regressão de Weibull (ver (9)) só com a covariável dia da semana (1 para segunda-feira e 0 para outros dias tem-se os seguintes estimadores de máxima verossimilhança para os parậmetros de regressão: $\beta_{0}$ e $\beta_{1}:\left(\hat{\beta}_{0}\right)=1,98837$ e $\left(\hat{\beta}_{1}\right)=0,286096$. Além disso o parâmetro de forma é estimado por $(\hat{\alpha})=$ 1,1084. Assim temos os estimadores de máxima verossimilhança (propriedade de invariância para os estimadores de máxima verossimilhança) para $\lambda$ $=\exp \left(\beta_{0}+\beta_{1}\right.$ dia-semana $)$ dados respectivamente por $\widehat{\lambda}=9,72273$ (segundas-feiras) e $\widehat{\lambda}=7,30362$ (outros dias da semana). Desta forma de (4), a Tabela 5 apresenta as probabilidades dos tempos entre chegadas serem maiores do que valores fixados $t^{*}$ para os diferentes dias da semana; observase que as probabilidades são bem menores para dias diferentes de segunda-feira. Isto ocorre, pois departamento de controle de qualidade não trabalha nos finais de semana, provocando um acúmulo de lotes (produzidos no final de semana) para inspeção na segunda-feira - o que justifica os tempos entre chegadas serem maiores neste dia da semana.

Tabela 5. Estimativas de $\hat{\boldsymbol{R}}\left(t^{*}\right)$ para alguns valores de $t^{*}$.

\begin{tabular}{|l|c|c|}
\hline $\mathrm{t}^{*}$ & $\begin{array}{c}\hat{\boldsymbol{R}}\left(\boldsymbol{t}^{*}\right) \\
\text { segundas-feiras }\end{array}$ & $\begin{array}{c}\hat{\boldsymbol{R}}\left(\boldsymbol{t}^{*}\right) \\
\text { outros dias semana }\end{array}$ \\
\hline 0,3208 & 0,977463 & 0,969183 \\
\hline 0,7078 & 0,946675 & 0,927513 \\
\hline 1,9705 & 0,843270 & 0,791299 \\
\hline 5,5372 & 0,585204 & 0,479157 \\
\hline 9,1597 & 0,392186 & 0,276568 \\
\hline 16,5417 & 0,164931 & 0,084185 \\
\hline 21,0263 & 0,095257 & 0,039616 \\
\hline
\end{tabular}

Fonte: própria.

Estes resultados foram de grande valia para os engenheiros e administradores da indústria, pois possibilitou uma modelagem do setor de controle 
de qualidade mais fidedigna para a realização de uma Simulação Discreta de Eventos. Em especial, foram determinados parâmetros mais adequados na criação de entidades (parâmetros de forma e escala da distribuição de Weibull para os tempos entre chegada) e na definição do calendário (dias da semana simulados: segunda-feira ou demais dias) na ferramenta utilizada.

Além disso, os resultados permitiram aos gestores um replanejamento da alocação de pessoas no departamento de controle da qualidade, visando uma melhor programação das inspeções segundo as covariáveis identificadas. Tal fato propiciou uma melhor utilização da capacidade produtiva, sem comprometer significativamente o lead-time médio dos testes de qualidade.

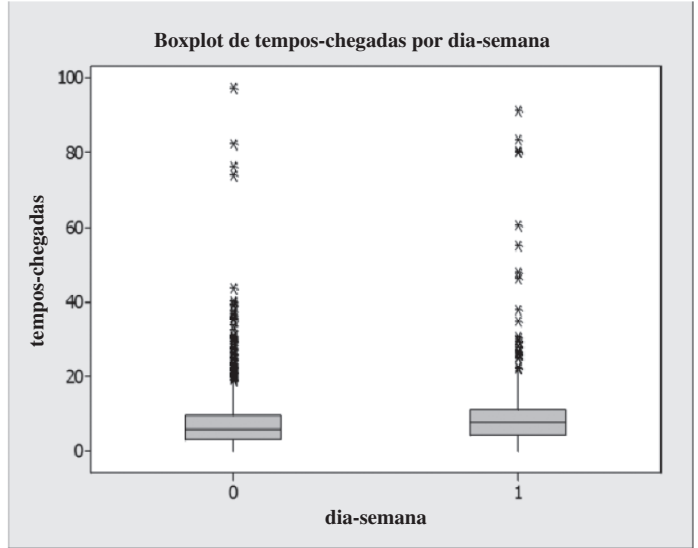

Figura 4. Box-plots para os tempos entre chegadas de lotes nas segundas-feiras (1) e outros dias da semana (0).

Fonte: própria.

Por fim, cabe ressaltar que a abordagem estatística realizada neste artigo pode ser empreendida em diversos outros tipos de sistemas produtivos, objetivando melhores análises e, consequentemente, melhores decisões.

\section{REFERÊNCIAS}

[1] D.R. Cox and V.I. Isham. "Point processes". Chapman and Hall. London, UK. 1976. ISBN: 0122264606180.

[2] S.M. Ross. "Stochastic processes". Wiley\&Sons. New York, USA. 1995. ISBN: 0471120626.

[3] D.L. Snyder and M.I. Miller. "Random point processes in time and space". Springer-Verlag.
New York, USA. 1991. ISBN: 0471810215 , 9780471810216.

[4] M.E. Kuhl, S.G. Sumant and J.R. Wilson. "An Automated Multiresolution Procedure for Modeling Complex Arrival Process". INFORMS Journal of Computing. Vol. 18, Issue 1, pp. 3-18. 2006. ISSN: 1526-5528 (Online). DOI: 10.1287/ijoc.1040.0113.

[5] P.A.C. Miguel. "Estudo de caso na engenharia de produção: estruturação e recomendações para sua condução". Produção. Vol. $17 \mathrm{~N}^{\circ} 1$, pp. 216-229. 2007. ISSN: 0103-6513 (online).

[6] J.W.M. Bertrand and J.C. Fransoo. "Operations management research methodologies using quantitative modeling". International Journal of Operations \& Production Management. Vol. 22, Issue 2, pp. 241-261. 2002. ISSN: 0144-3577.

[7] E.M. Lakatos y M.A. Marconi. "Fundamentos de metodologia científica". Editora Atlas. $6^{\text {ta }}$ edición. São Paulo, Brasil. 2008. ISBN: 978-85-224-4015-3.

[8] A.C. Gil. "Como elaborar projetos de pesquisa". Editora Atlas. $5^{\text {ta }}$ edición. São Paulo, Brasil. 2010. ISBN: 85-224-5823-5.

[9] D. Gross and C.M. Harris. "Fundamentals of queueing theory". John Wiley \& Sons. New York, USA. 1974. ISBN: 0-471-32812-X.

[10] L. Kleinrock. "Queueing systems". John Wiley \& Sons. New York, USA. Vol. 1, pp. 1-417. 1975. ISBN: 0471491101.

[11] R. Larson and A. Odoni. "Urban Operations Research". Prentice Hall Inc. Englewood Cliffs. New Jersey, USA. 1981. ISBN: 0-13-939447-8.

[12] Y.W. Shin and C.E.M. Pearce. "The BMAP/G/1 vacation queue with queue-length dependent vacation schedule". Journal of the Australian Mathematical Society Serie B. Vol. 40, pp. 207-221. 1998.

[13] M.C. Ausín, M.P. Wiper and R.E. Lillo. "Bayesian estimation for the M/G/1 queue using a phase-type approximation". Journal of Statistical Planning and Inference. Vol. 118, Issues 1-2, pp. 83-101. 2004. ISSN: 0378-3758.

[14] M.C. Ausín, M.P. Wiper and R.E. Lillo. "Bayesian prediction of the transient behaviour and busy period in short- and longtailed GI/G/1 queueing systems". Statistics Computational Statistics \& Data Analysis. 
Vol. 52, pp. 1615-1635. 2008. ISSN: 01679473. DOI: $10.1016 /$ j.csda.2007.05.009.

[15] K. Wu and K. Hui. "The Determination and Indetermination of Service Times in Manufacturing Systems". IEEE Transactions on Semiconductor Manufacturing. Vol. 21, Issue 1, pp. 72-82. 2008. ISSN: 0894-6507.

[16] A.A. Emadzadeh and J.L. Speyer. "On Modeling and Pulse Phase Estimation of X-Ray Pulsars". IEEE Transactions on Signal Processing. Vol. 58, Issue 9, pp. 4484-4495. 2010. ISSN: 1053-587X.

[17] G. Pang and W. Whitt. "Two-parameter heavy-traffic limits for infinite-server queues". Queueing Systems. Vol. 65, pp. 325-364. 2010. ISSN: 1572-9443 (Online). DOI: 10.1007/s11134-012-9303-0.

[18] M.E. Zonderland, R.J. Boucherie, N. Litvak and C.L.A.M. Vleggeert-Lankamp. "Planning and Scheduling of semi-urgent surgeries". Health Care Management Science. Vol. 13, pp. 256-267. 2010. ISSN: 1572-9389 (Online). DOI: 10.1007/s10729-010-9127-6.

[19] W. Liu and W. Whitt. "Large-time asymptotics for the Gt/Mt/st +GIt many-server fluid queue with abandonment". Queueing Systems. Vol. 67, pp. 145-182. 2011. ISSN: 1572-9443 (Online). DOI: 10.1007/s11134-010-9208-8.

[20] M.S. Stephens and W.I. Ukpere. "Airport capacity utilization in Nigeria: A performance and efficiency analysis". African Journal of Business Management. Vol. 5, Issue 27, pp. 11104-11115. 2011. ISSN: 1993-8233 (Online). DOI: 10.5897/AJBM11.1007.

[21] M. PIDD. "Computer Simulation in Management Science". John Wiley Prof. $5^{\text {th }}$ edition. Lancaster, United Kingdom. 2004. ISBN-10: 0470092300.

[22] W.D. Kelton, R.P. Sadowski and D.T. Sturrock. "Simulation with Arena". McGraw- Hill. Fourth edition. New York, USA. 2007. ISBN: 0072919817.

[23] C.R. Harrell, B.K. Ghosh and R. Bowden. "Simulation Using ProModel". McGrawHill Professional. Third edition. USA. 2011. ISBN-10: 0073401307.

[24] N. Sakurada e D.I. Miyake. "Aplicação de simuladores de eventos discretos no processo de modelagem de sistemas de operações de serviços". Gestão \& Produção. Vol. $16 \mathrm{~N}^{\circ} 1$, pp. 25-43. 2009. ISSN: 0104-530X (Online).
[25] W. Weibull. "A statistical distribution function of wide applicability". Journal of Applied Mechanics - ASMC. Vol. 18, Issue 3, pp. 293-297. 1951.

[26] N.L. Johnson, S. Kotz and N. Balakrishnan. "Continuous univariate distributions". Wiley $\&$ Sons. Wiley series in probability and mathematical statistics: applied probability and statistics. $2^{\text {nd }}$ edition. New York, USA. Vol. 1. 1994. ISBN: 0-471-58494-0.

[27] W. Nelson. "Applied life data analysis". Wiley. New York, USA. 2004. ISBN: 0-471-64462-5.

[28] E. Colosimo e S.R. Giolo. "Análise de sobrevivência aplicada". Edgard Blucher. São Paulo, Brasil. 2006. ISBN: 3-900051-07-0.

[29] J. Achcar, R.S. Brookmeyer and W.G. Hunter. "An application of Bayesian analysis to medical follow-up data". Statistics in Medicine. Vol. 4, pp. 509-520. 1985. ISSN: 1097-0258 (Online). DOI: 10.1002/sim.4780040411.

[30] D.N.P. Murthy, M. Bulmer and J.A. Eccleston. "Weibull model selection for reliability modeling". Reliability Engineering \& System Safety. Vol. 86, pp. 257-267. April, 2004. ISSN: 0951-8320.

[31] L.Attardi, M. Guida and G.A. Pulcini. "Mixed-Weibull regression model for the analysis of automotive warranty data". Reliability Engineering \& System Safety. Vol. 87, pp. 265-273. February, 2005. ISSN: 0951-8320.

[32] A. Kleyner and P.A. Sandborn. "A warranty forecasting model based on piecewise statistical distributions and stochastic simulation". Reliability Engineering \& System Safety. Vol. 88, pp. 207-214. 2005. ISSN: 0951-8320.

[33] A.S. Babuy and V. Jayabalan. "Weibull Probability Model for Fracture Strength of Aluminium (1101)-Alumina Particle Reinforced Metal Matrix Composite". Journal of Material Science Technology. Vol. 25, Issue 3, pp. 341-343. 2009. ISSN: 1005-0302.

[34] W.Q. Meeker and L.A. Escobar. "Statistical methods for reliability data". Wiley\&Sons. New York, USA. 1998. ISBN: 0471143286.

[35] M. Abramowitz and I.A. Stegun. "Handbook of mathematical functions with formulas, graphs and mathematical tables". Dover 
Publications Inc. 9th edition. New York, USA. 1972. ISBN: 0-486-61272-4.

[36] D.C. Montgomery and G.C. Runger. "Applied statistics and probability for engineers". Wiley \& Sons. $5^{\text {th }}$ edition. New York, USA. 2010. ISBN: 0-471-74589-8.

[37] J.F. Lawless. "Statistical models and methods for lifetime data, wiley series in probability and mathematical statistics". Wiley \& Sons. New York, USA. 1982. ISBN-10: 0471085448.

[38] E.J. Gumbel. "Statistical theory of extreme values and some practical applications". Applied Mathematics Series. Vol. 33. United States. U.S. Govt. Department of Commerce, National Bureau of Standards. 1954. 